\title{
The physics of a popsicle stick bomb
}

Jérémy Sautel, Andréane Bourges, Aude Caussarieu, Nicolas Plihon, and Nicolas Taberlet* Univ Lyon, Ens de Lyon, Univ Claude Bernard, CNRS,

Laboratoire de Physique, Département de Physique, F-69342 Lyon, France

(Dated: December 13, 2019)

Popsicle sticks can be interlocked in the so-called cobra weave to form a chain under tension. When one end of the chain is released the sticks rapidly disentangle, forming a traveling wave that propagates down the chain. In this paper the properties of the traveling front are studied experimentally and classical results from the theory of elasticity allow for a seemingly impossible dimensional analysis. The study presented here can help undergraduate students familiarize with experimental techniques of image processing. Most importantly it demonstrates the predicting power of dimensional analysis and scaling laws.

\section{INTRODUCTION}

\section{A. What is a popsicle stick bomb?}

Wooden popsicle sticks can be bent and interlocked into a pattern known as the "cobra weave" (due to the resemblance with the patterns formed by the scales of an actual cobra), see figure 1 . In the displayed setup, each individual stick is bent to rest alternatively on top of and under four perpendicular sticks (as shown at the bottom of figure 2) but other geometrical configurations could also be woven. When weaving the chain one realizes that a large potential energy is stored into the sticks and the ends of the chain have to be held still. When the weight holding the sticks down on one end of the chain is removed, the so-called stick bomb detonates: the sticks pop-up and a traveling wave is formed.

This system is reminiscent of similar mechanical chain reactions in a line of dominoes ${ }^{1-3}$, or in an assembly of mousetraps ${ }^{4,5}$, both systems displaying propagation fronts and conversion of the stored potential energy (whether gravitational or elastic) into kinetic energy. This stick bomb also involves a competition between elastic and gravitational energy. This interesting concept is present in many domains of physics, from the well-known problem of the bounce of a ball, involving mechanical physics $^{6,7}$, to the realization of a precise measurement of the gravity constant by making ultra-cold atoms bounce on an atomic mirror, in quantum mechanics ${ }^{8}$. In this article the speed of the traveling wave and the height reached by individual sticks are studied through simple hands-on experiments.

\section{B. International Physicists Tournament}

The work presented here was done in preparation for the International Physicists Tournament (http:// iptnet.info/), a world-wide competition for undergraduate students. Each national team is composed of six students who work throughout the academic year on a list of seventeen open questions and present their findings during the tournament.
Unlike the typical physics exam, the problems must not only be presented, but also challenged and reviewed by the other participants, allowing students to respectively assume the roles of researchers, referees and editors. In addition to the challenge that the tournament represents, it provides students with an exciting and eye-opening experience in which they learn how to design experiments with the aim of solving physics problems, and to constructively criticize scientific solutions.

The authors would highly recommend participation in the IPT as a rare learning opportunity for undergraduate students.

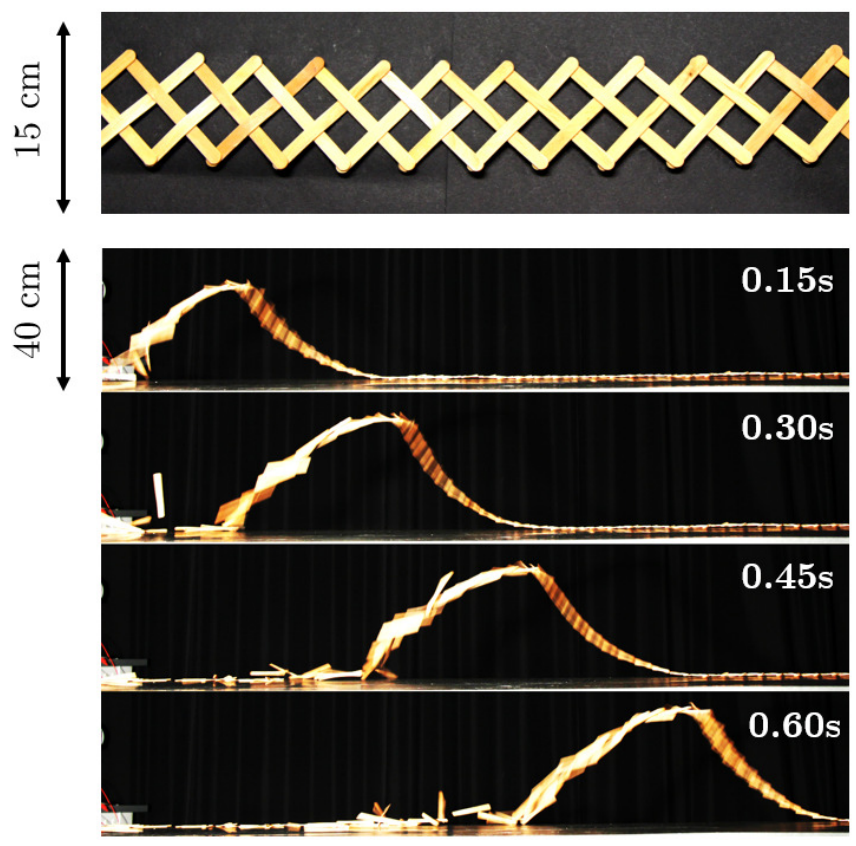

FIG. 1. Top: top-view of the popsicle sticks interlocked in the cobra weave. Bottom: side-view showing the wave propagating. 


\section{What undergraduate students can learn from this problem}

This problem can provide an amusing introduction to solid mechanics, giving a simple example of the conversion of stored elastic energy into kinetic and gravitational potential energy ${ }^{9}$ in which students can measure an energetic yield and consider sources of dissipation. The problem is also an interesting illustration of the theory of elasticity since the study of the statics and dynamics of a single bent stick fits in the Euler-Bernoulli theory for beam deflection ${ }^{10-12}$. Moreover, students may familiarize with experimental techniques such as particle tracking or with the interpretation of a space-time diagram ${ }^{13}$. Finally, this paper may help undergraduate students grasp the importance of dimensional analysis ${ }^{14-16}$ as well as the predicting power of scaling laws ${ }^{17,18}$.

\section{Outline}

The aim of this study is to understand the dependence of the velocity and of the height of the cobra wave on the parameters of the popsicles and of the weaving. In a nutshell, section II examines the elasticity of one unique stick, section III focuses on the traveling wave obtained with one given set of identical sticks, while the properties of the sticks (dimensions and material) are varied in section IV.

To model the popsicle stick bomb and understand why a wave propagates, sticks are modeled by beams in the theory of elasticity framework whose important results are recalled in section II. We will first focus on the static aspects by considering the mechanical equations, boundary conditions and the energy stored in a beam. The dynamics of a stick will also be discussed for a clamped beam, by studying the frequency (in the linear approximation) of the first transverse vibration mode. We then explain how the parameters of the sticks are measured and discuss the efficiency of the conversion of stored elastic energy into kinetic and gravitational energy.

Experimental data obtained for the traveling waves using a given set of wooden sticks will be exposed in part III where the experimental methods used to measure the speed and height of the traveling wave are explained.

Finally, we will derive in part IV scaling laws allowing us to predict the speed and height of a cobra wave, by knowing only some intensive (Young's modulus, density) and extensive (geometrical dimensions) parameters of one stick. The predicting power of the scaling law and the influence of dimensionless parameters are discussed.

\section{ELASTICITY OF A SINGLE STICK}

\section{A. Euler-Bernoulli beam theory}

This section analyses the elastic properties of one single stick. Unless otherwise mentioned the classical results presented in this paragraph are taken from "The Theory of Elasticity" by Landau and coworkers ${ }^{10}$.

a. General hypotheses The Euler-Bernoulli beam theory is a simplification of the theory of elasticity limited to small deformations and in which the shear stress in neglected. In this framework there exists a neutral axis along the beam whose length remains constant and to which the cross section remains perpendicular. In the case where the beam is only constrained by point supports (i.e. with no distributed load or torque) the static beam equation $z(x)$ follows:

$$
\frac{d^{4} z}{d x^{4}}=0
$$

meaning that between two consecutive contact points, the equation of the beam is a third degree polynomial whose coefficients are given by the boundary conditions.

Equation 1 is derived analytically but a hand-waving argument can help understand its fundamental physical meaning. The local curvature $c=\frac{d^{2} z}{d x^{2}}$ of the stick is the first relevant derivative of the profile $z(x)$ that plays a crucial role. Indeed, a stick can be translated (affecting the absolute value of $z$ ) or rotated (changing $\left.\frac{d z}{d x}\right)$ without any changes to the physics of the bending (except for the unrealistic case of extremely flexible sticks whose shape is affected by gravity). In terms of curvature, equation 1 therefore simply reads: $\frac{d^{2} c}{d x^{2}}=0$.

b. Elastic potential energy A point contact at a given position $x_{0}$ simply imposes the position $z\left(x_{0}\right)$ and continuity of the first and second derivatives of $z$. Note that the force locally imposed by the point contact can be computed from the third derivative of $z$. More importantly for the present article, the elastic potential energy stored in the bent beam is given by:

$$
U_{e l}=\int_{0}^{L} \frac{1}{2} E I\left(\frac{d^{2} z}{d x^{2}}\right)^{2} d x
$$

where $E$ is the Young's modulus of the material and $I$ the second moment of area, given by $I=l h^{3} / 12$ for a rectangular beam of constant section $l \times h$ and length $L$. Again, this equation can be understood in term of the curvature $c$ of the sticks: $U_{e l} \propto 1 / 2 \int c^{2} d x$.

The elastic potential energy is given in equation 2 for various configurations: deflection of $\delta$ at the end of a clamped beam (or cantilever), at the center of a 2-point bent beam $(x=0$ and $x=L)$, for a 4 -point bent beam 


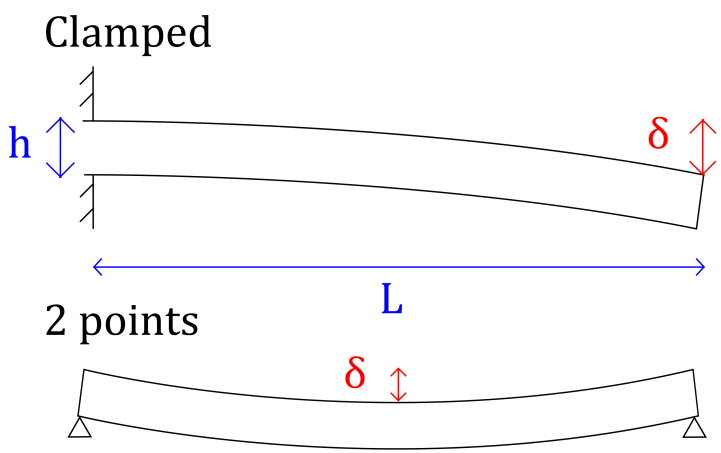

4 points

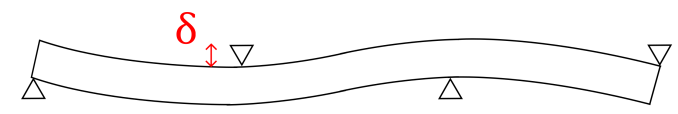

4 sticks

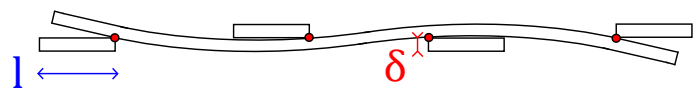

FIG. 2. Sketches of a single stick in four configurations. For clarity the thickness, $h$, and the deformation, $\delta$, are largely exaggerated.

$(x=0, L / 3,2 L / 3$ and $L)$, and for a beam woven between four perpendicular sticks. Note that the last case requires a careful analysis since one has to determine on which edges of the central sticks the beam rests (the contact points are indicated by dots in figure 2).

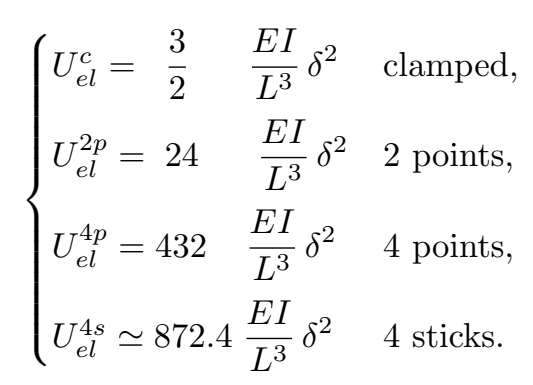

The numerical prefactor in the last equation is obtained using the dimensions of the sticks shown on figure $1 ; l=10 \mathrm{~mm}$ and $L=110 \mathrm{~mm}$. Indeed, when taking the width of the sticks, $l$, into account, one realizes that the first obvious effect is that their length is reduced by $2 l$ since the outermost contact points are at a distance $l$ from the ends. Moreover the width, $l$, also induces a larger deformation and hence a greater elastic energy in the central part of the bent stick. The energy was computed using Maple for various values of $l / L$ and we empirically found that accounting for $l$ is equivalent to reducing its length to $L-2.3 l$, meaning that the elastic potential energy is given by :

$$
U_{e l}^{4 s}(l / L)=U_{e l}^{4 p} \frac{1}{(1-2.3 l / L)^{3}} \equiv U_{e l}^{4 p} f(l / L) .
$$

The prefactor in the last line of equation 2 is then given by: $872.4 \simeq 432 f(10 / 110)$.

c. Vibrations The equation of motion describing the dynamics of a uniform beam of mass $m$ undergoing transverse deformations (forbidding any twisting) can be easily derived from the lagrangian of a stick and is given by $^{10}$ :

$$
E I \frac{\partial^{4} z}{\partial x^{4}}+\frac{m}{L} \frac{\partial^{2} z}{\partial t^{2}}=0
$$

Solving equation 4 allows one to compute the eigen modes of a vibrating beam and the corresponding frequencies. For instance, the angular frequencies of the eigen modes of a beam clamped at one end (cantilever) are given by $\omega_{n}=\alpha_{n} \sqrt{\frac{E I}{m L^{3}}}$ with $\alpha_{1} \simeq 3.52$, $\alpha_{2} \simeq 22.0, \alpha_{3} \simeq 61.7$, when for a free beam (under no constrants): $\alpha_{1} \simeq 22.4, \alpha_{2} \simeq 61.7, \alpha_{3} \simeq 121.0$. Note again that all the eigen frequencies are proportional to a characteristic frequency $\omega^{*} \equiv \sqrt{\frac{E I}{m L^{3}}}$ with a geometrical prefactor that depends on the boundary conditions. It is worth mentioning that $\omega^{*}$ can be easily deduced from a dimensional analysis of equation 4 .

d. Equivalent spring-mass system Obviously the energy stored varies widely from one configuration to another but the reader must note that the elastic potential energy is always proportional to a characteristic energy $\frac{1}{2} k \delta^{2}$, where $k \propto E I / L^{3}$, with a geometrical prefactor. The characteristic energy can be regarded as the potential energy stored in a linear spring. A dimensional analysis seems in principle impossible because of the large number of lengths in the problem (length, width and thickness of the beam, pitch of the pattern). However the theory of elasticity provides one with an effective spring constant which then allows for a dimensional analysis. A characteristic elastic energy stored in a stick in cobra weave is therefore given by $U_{e l}^{*} \equiv \frac{E l h^{5}}{L^{3}}$ since in this case, the deflection is given by: $\delta=h$.

Even more interestingly for the scaling laws discussed in section IV, one can notice that $\omega^{*} \propto \sqrt{k / m}$, the angular frequency of a linear spring-mass system. Although seemingly impossible, a dimensional analysis of the problem is rendered possible by the theory of elasticity. Having understood this subtle point, one finds itself equipped with a characteristic time for the deformation of the beam woven in the cobra pattern: $T^{*}=\sqrt{m / k}$.

Note moreover that this characteristic time $T^{*}$ can be written as a function of the material properties (Young's modulus, $E$, and density, $\rho$ ):

$$
T^{*}=\frac{L}{h} L \sqrt{\frac{\rho}{E}}=\frac{L}{h} \frac{L}{c_{0}} .
$$

where the ratio $c_{0}=\sqrt{E / \rho}$ has the dimension of a velocity and is the speed of sound of a material whose Poisson's ratio, $\nu$, equals zero. For the more realistic value of 
$\nu=0.3$ for hard wood, the speed of sound (compression waves) is given by $\sqrt{\frac{E}{\rho} \frac{1-\nu}{(1+\nu)(1-2 \nu)}} \simeq 1.16 c_{0}$.

Interestingly, the characteristic time can therefore be seen as the product of the length-to-thickness aspect ratio of the sticks, $L / h$, and the time it takes a sound wave to propagate along the stick.

\section{B. Measurement of the mechanical parameters}

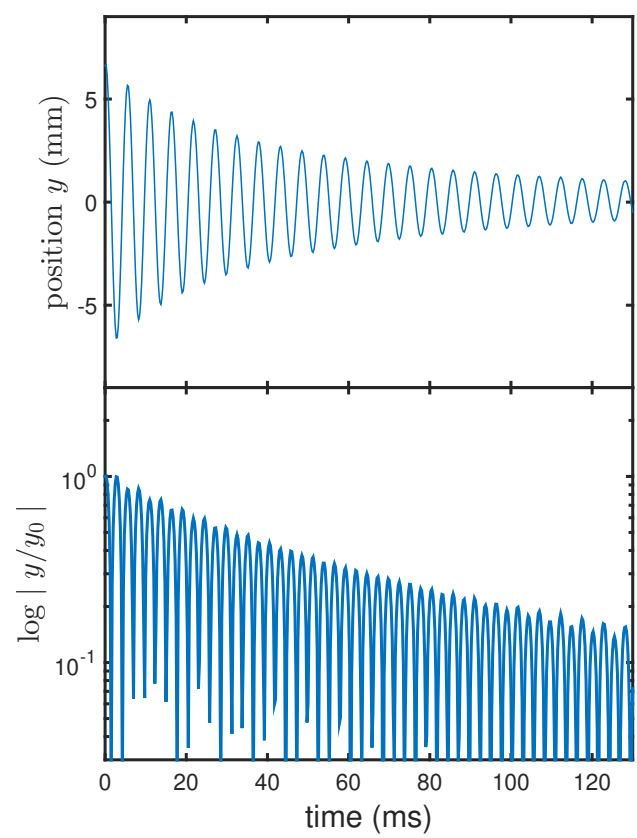

FIG. 3. Free oscillations of the free end of a clamped wooden stick (Wood 2 in table I) in linear (top) and log (bottom) scales. Measuring the frequency leads to the value of the Young's modulus of the stick. The damping is caused by internal dissipation within the stick.

Mass and dimensions of the sticks can be easily measured using a scale and a caliper. The Young's modulus, $E$, or equivalently, the effective spring constant, $k$, were measured using two separate methods on a beam clamped at one end. The first method that was used consists in simultaneously measuring the force, $F$, applied at the free end of the stick (using a dynamometer) and the resulting deflection $\delta$. The relation between the two is given by the Euler-Bernoulli theory: $F=3 E I \delta / L^{3}$. We have checked that the relation between the force and the deflection remains linear for small deflections $(\delta<L / 10)$ and the slope gives a direct measurement of the effective spring constant, which leads to the value of the Young's modulus.

The second method consists in studying the first mode of vibration of a clamped stick. The frequency of this mode is given in the previous paragraph and allows one to compute the Young's modulus (or equivalently the effective spring constant). This second method is far more accurate than the first method. Figure 3 shows the position of the free end of a wooden stick of length $L=85$ $\mathrm{mm}$ after it was given an initial deflection of $\delta=5 \mathrm{~mm}$. The data was obtained by tracking the position of the end of the stick using the "Analyze Particle" tool in ImageJ, a free software developed by NIH, from a film taken at 1000 FPS with a resolution of $10 \times 1500$ pixels.

It is worth noting that the vibrations of the stick are rapidly damped. In principle the damping can originate both from air resistance (drag) and from internal dissipation. A rapid estimation of the drag force (based on the Reynolds number) shows that the effective quality factor of the vibrations should be of the order of $10^{6}$. It is very clear from figure 3 that the damping is much faster (the amplitude is halved in less than 10 oscillations), which indicates that the main source of damping is the internal dissipation within the stick, whose influence will be discussed in section IV. Although this dissipation mechanism is not expected to be linear, the damping is remarkably close to an exponential decay (see figure 3 ).

\section{Energy release and efficiency}

In this section we will discuss the efficiency of the conversion of elastic potential energy into gravitational energy.

To measure this ratio we performed a very simple experiment, considering the case of a stick which is stuck between two nails, as shown on figure 4 and bent when one applies a force $F$, with the help of a dynamometer, at the free end of the stick. The energy stored into the stick is not computed from the theory (which relies on a number of assumptions) but instead directly measured as the work provided by the dynamometer: $U_{e l}=\int_{0}^{\delta} F d \delta=\frac{1}{2} F \delta$.

When the dynamometer is removed the stick rises, reaches a maximum point, and then falls down. We measure the maximum height $H$ reached by the center of mass of the stick, in order to compute the maximum gravitational energy $E_{g}=m g H$. Repeating this experiment for several values of $F$, one is able to plot $E_{g}$ as a function of $U_{e l}$ in order to determine the efficiency of the conversion of elastic energy into gravitational energy. The plot is represented on figure 4, and gives a constant efficiency of roughly 0.25 . Only a fraction of the available energy is therefore used to reach the maximum height. In this particular case $75 \%$ of the energy is converted into vibrations (mostly transverse but possibly in twisting modes as well) and rotation (in all directions) or dissipated (because of air friction or internal dissipation).

This empirical efficiency of $25 \%$ is specific to the setup used here and one should therefore expect a different efficiency for a stick in the cobra weave. The simple geometry presented in this section optimizes the height reached 


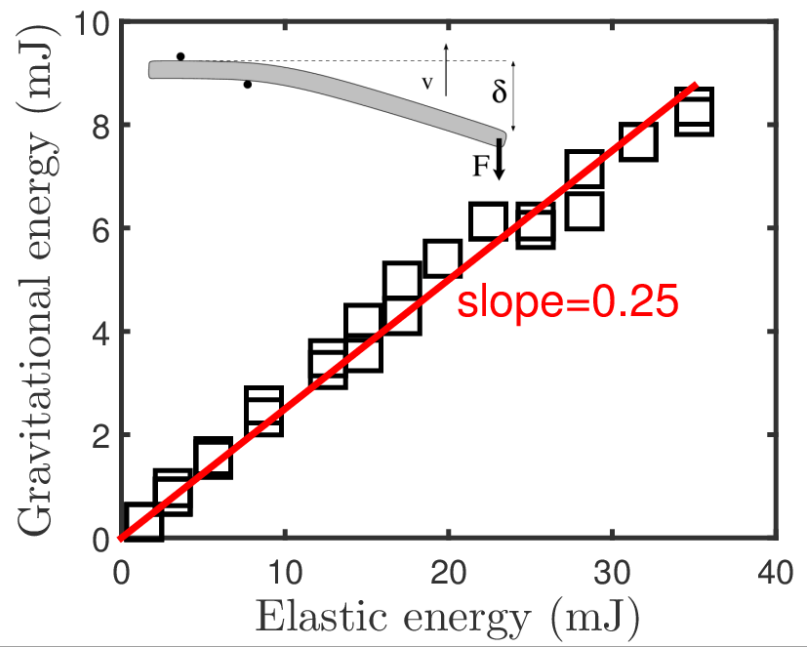

FIG. 4. Gravitational energy of a stick as a function of the elastic energy stored, in the case of the simple setup shown on the upper part of the figure. A stick is placed between two nails and bent at its free end. When the force is released, the sticks takes off vertically. The data shows a remarkably linear behavior which defines a constant conversion efficiency of roughly $25 \%$.

by a stick whereas a more symmetrical shape, such as the cobra weave (see figure 2) would favor vibrations at the expenses of the height. Moreover, in the cobra weave solid friction between the sticks can be very high, and is another source of energy dissipation. Hence, the efficiency of the conversion of elastic energy into gravitational energy for an actual popsicle stick bomb is expected to be even lower than $25 \%$. For the sticks used in figure 7 the efficiency was found to be close to $5 \%$.

\section{SPEED AND HEIGHT OF THE WAVE FOR ONE SET OF STICKS}

The height and velocity of the wave may depend on numerous parameters. The material properties and the dimensions of the sticks are discussed in section IV. Here instead we focus on results obtained with a given set of wooden sticks, varying only the pitch of the cobra pattern.

\section{A. Experimental methods}

A 3-m long chain of sticks is woven keeping the extremities clamped using large weights. When the weight is removed at one end, the stick bomb detonates and the propagation of the wave is filmed using a fast camera at rates up to $2000 \mathrm{FPS}$ with a resolution of $1500 \times 10$ pixels.

The films obtained were processed using the Orthogonal View function in ImageJ, in order to perform a spatiotemporal analysis. This function consists in choosing one fixed horizontal line for all the pictures located approximately at half-height of the wave. A threshold is applied to make the picture black and white. The profile along this line is then plotted for all the frames which therefore creates space-time diagram (see figure 5 ).

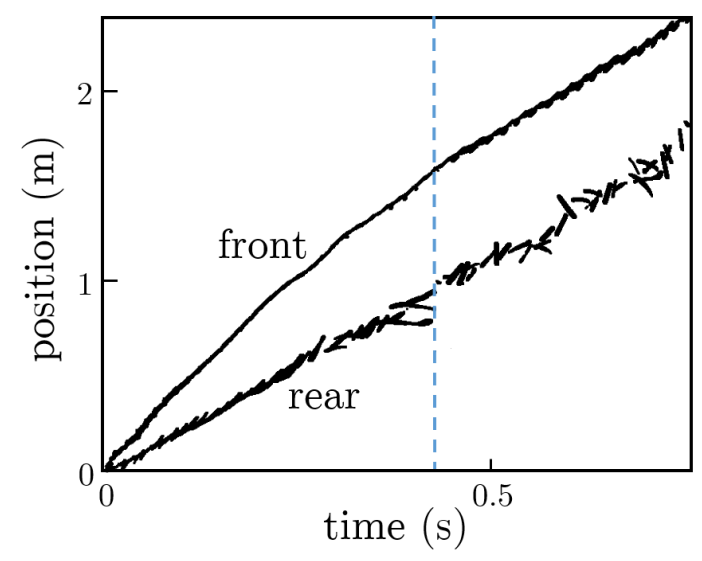

FIG. 5. Space-time diagram of the wave using wet wooden sticks. The two lines can be interpreted as the horizontal position of the ascending (front) and descending (rear) of the "hump" seen on figure 1, measured at half-height. A steady state is reached after the front has traveled $1.5 \mathrm{~m}$ (dashed line), corresponding to roughly 100 sticks.

When the chain reaction is triggered at one end of the chain a traveling wave is triggered. One can easily see the evolution of the front and rear position of the wave as a function of time. A steady state, in which the half width of the wave remains constant and the trajectory of the front is a straight line, is reached after the front has traveled for no less than $1.5 \mathrm{~m}$ (dashed line). It is therefore important that the length of the chain is longer than this transient length (which may depend on the type of sticks used).

The velocity is measured as the slope of the trajectory in the steady regime. The height of the wave is directly measured on the frames (see figure 1), considering for each of them the altitude reached by the center of mass of a highest stick. The results are averaged over the steady state.

The authors need to mention that for convenience the sticks used in figures 1 and 5 were made of remarkably flexible wood and were soaked in water for several days. This results in low speed $(1.7 \mathrm{~m} / \mathrm{s})$ which helps taking sharp images and produces clear space-time plots. However in the following more rigid sticks are used.

\section{B. Results and discussion}

The results presented in this section were obtained using one set of rigid sticks (Wood 2 in table I), soaked in water for three weeks (thickness $h=2.3 \mathrm{~mm}$, width $l=10 \mathrm{~mm}$, length $L=114 \mathrm{~mm}$, density $\rho=970 \mathrm{~kg} \cdot \mathrm{m}^{-3}$ 
and Young's modulus $E=5.0 \mathrm{GPa}$, measured using the method presented in section IIB).

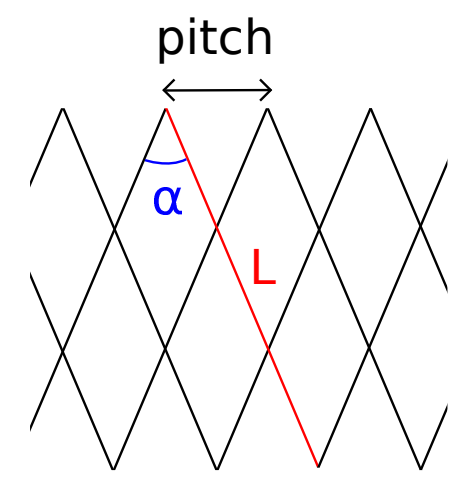

FIG. 6. Sketch of the cobra weave: the black lines correspond to the sticks of length $L$. The pitch is defined as the distance between the extremities of two consecutive sticks: pitch $=$ $\frac{2 L}{3} \sin \frac{\alpha}{2}$.

This section is devoted to the study of the influence of the pitch of the pattern (see figure 6) on the speed and height of the wave. The pitch appears to be a crucial parameter and can be easily changed by varying the angles between the sticks when building the chain. One can easily show that this pitch is given by pitch $=\frac{2 L}{3} \sin (\alpha / 2)$ where $\alpha$ is the angle between the sticks (see figure 6 ). The same set of sticks was used to build several chains with different values of $\alpha$, and the results of this study are shown on figure 7 .

The first conclusion that can be drawn is that the height is unaffected when the pitch is varied whereas the speed of the wave clearly increases with increasing pitch. As discussed in section II for the simpler case of a single stick, the height reached by the wave finds its origin in the elastic energy stored when the chain is built. In a steady state each individual stick can on average only hope to recover its own elastic energy. When neglecting both the width $l$ of the bent stick (i.e. considering $2 \mathrm{D}$ cross sections as in figure 2) and the twisting of the sticks, the elastic energy stored in the bending is independent of the pitch. This leads to a constant height when the pitch is varied. The data presented on figure 7 also enables one to estimate the efficiency of the energy conversion. On average, the efficiency is roughly $5 \%$, considerably less that the $25 \%$ obtained with the more favorable setup of figure 4 .

As discussed in section II the motion of a stick occurs over a typical time $T^{*}$, specific to each set of sticks, with numerical prefactors that depend on the exact configuration. For a given set of sticks, when the pitch is varied, a dimensional analysis therefore indicates that the speed of the wave should scale as the ratio: pitch $/ T^{*}$. This predicted linear dependence of the velocity as a function of the pitch is indeed experimentally confirmed (blue line on figure 7 ). For the sake of clarity, the width $l$ of the sticks is not shown on figure 6 but clearly, the pitch has to exceed this value. Therefore, the linear fit is obtained using an offset of $l=10 \mathrm{~mm}$.

In conclusion, a dimensional argument allows one to predict both the linear dependence of the speed and the constant height as a function of the pitch.

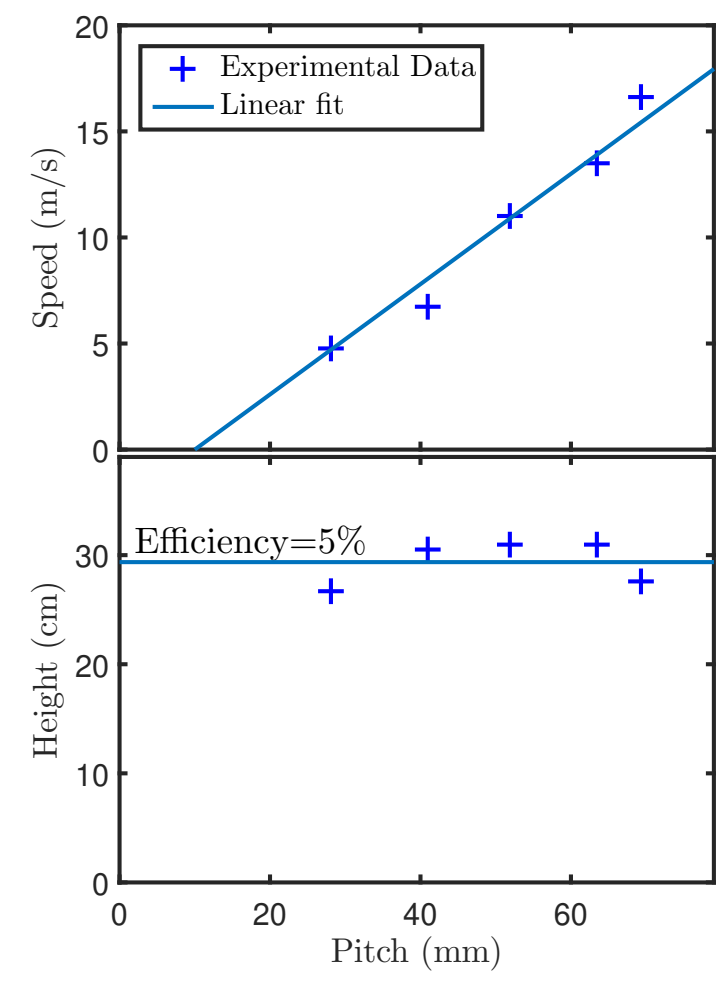

FIG. 7. Dependence of the speed (top) and height (bottom) of the wave on the pitch of the pattern. The speed $V$ depends linearly on the pitch, however with an offset corresponding to the width of the sticks $l$. The height $H$ seems to be independent of the pitch. The blue line indicates an efficiency of $5 \%$.

\section{SCALING LAWS}

\section{A. Various materials and dimensions}

The previous section presented data obtained with the same wooden sticks as the pitch of the cobra weave was varied. In addition to these experiments the dimensions and material of the sticks were varied (table I gives a summary of the sticks used) while the angle $\alpha$ of the cobra weave is kept constant (to $90^{\circ}$ ). In order to vary their Young's modulus, the wooden sticks were soaked in water, for durations ranging from a few days to a few weeks. This process did make the wooden sticks less rigid (their Young's modulus was computed in a simple clamped configuration using the method presented in section II B) but it also affected their density (up to a factor 2 ) and size (with an increase up to 10\%). For all sets 
of sticks the Young's modulus $E$ and density $\rho$ were independently measured prior to any experiment and the corresponding so-called speed of sound $c_{0}=\sqrt{E / \rho}$ is reported in table I. Plastic sticks of various sizes, made of PVC (polyvinyl chloride), were also used.

TABLE I. Parameters of sticks

\begin{tabular}{lcccc}
\hline \hline Name & $\begin{array}{c}\mathrm{h}(\mathrm{mm}) \\
\pm 0.1\end{array}$ & $\begin{array}{c}\mathrm{l}(\mathrm{mm}) \\
\pm 0.1\end{array}$ & $\begin{array}{c}\mathrm{L}(\mathrm{mm}) \\
\pm 1\end{array}$ & $\begin{array}{c}c_{0}(\mathrm{~m} / \mathrm{s}) \\
\pm 100\end{array}$ \\
\hline Wood 1 & 2.2 & 9.5 & 113 & 4600 \\
Wood 2 & 2.4 & 10.0 & 114 & 2000 \\
Wood 3 & 2.3 & 10.0 & 114 & 2300 \\
Wood 4 & 1.1 & 5.3 & 136 & 6500 \\
PVC 1 & 2.0 & 9.7 & 100 & 1400 \\
PVC 2 & 2.0 & 9.7 & 120 & 1400 \\
PVC 3 & 2.0 & 9.7 & 140 & 1400 \\
PVC 4 & 2.0 & 9.7 & 133 & 1400 \\
\hline \hline
\end{tabular}

Figure 8 shows the experimental results obtained using these various sticks plotting both the height, $H$, and velocity, $V$, of the wave. The relation between $H$ and $V$ is not expected to be simple and the data are not expected to fall onto a master curve. The figure is only intended as a synthetic way to present the experimental results. However a general trend can be seen as the stiffer and lighter sticks tend to both travel faster and reach a greater height, but the next paragraph reveals that thickness-to-length aspect ratio $h / L$ plays a crucial role.

\section{B. Characteristic velocity and height}

As shown in section III, the velocity of the wave is proportional to the pitch of the pattern divided by the characteristic time of vibration of a stick. Therefore a relevant characteristic velocity can be defined as pitch $/ T^{*} \propto L \sqrt{k / m} \propto \sqrt{E / \rho}(h / L) \equiv V^{*}=c_{0} h / L$. While the linear dependence on the pitch was confirmed in section III, the more complex dependence on the material parameters through the speed of sound $c_{0}$ and on the aspect ratio $h / L$ is examined in next paragraph. It is important to emphasize that the characteric speed, $V^{*}$, is not a theoretical prediction of the actual speed since unknown numerical prefactors have been left out.

Obviously the gravitational potential energy, $E_{g}=$ $m g H$, gained by a single stick finds its origin in the elastic energy stored during the weaving. Comparing these two energies is equivalent to comparing the height $H$ with a characteristic height defined as $H^{*} \equiv U_{e l} / m g=$ $\frac{c_{0}^{2}}{g}(h / L)^{4}$. The characteristic height, $H^{*}$, can then be seen as the maximum average height reach by the sticks if all the potential energy were converted into gravitational energy. Again, the predicted dependence of the height on the material parameters and dimensions of the sticks requires an experimental validation.

To summarize we have identified both a characteristic horizontal velocity and a characteristic height of the traveling wave:

$$
\left\{\begin{array}{l}
V^{*}=c_{0} \frac{h}{L} \\
H^{*}=36 f\left(\frac{l}{L}\right) \frac{c_{0}^{2}}{g}\left(\frac{h}{L}\right)^{4}
\end{array}\right.
$$

The two scales are specific to each set of sticks and depend solely on their speed of sound and aspect ratio. For dry wood the speed of sound is of the order of 4000 $\mathrm{m} / \mathrm{s}$ and the aspect ratio of the wooden popsicle sticks is $h / L=2 / 110$, leading to a characteristic velocity for the wave of $V^{*} \simeq 70 \mathrm{~m} / \mathrm{s}$, which gives the right order of magnitude (see figure 8). The characteristic height is $H^{*} \simeq 5.7 \mathrm{~m}$, which again gives the right order of magnitude given the low efficiency of the energy conversion (see figure 7 ).

\section{Collapse}

As one can see on figure 8, the experimentally gathered data displays an important spreading of the values of $V$ and $H$ between the different types of sticks used. Namely, the speed ranges from $7 \mathrm{~m} / \mathrm{s}$ to $36 \mathrm{~m} / \mathrm{s}$ and the height from $15 \mathrm{~cm}$ to $100 \mathrm{~cm}$. When the same data is rescaled using the characteristic quantities defined in the previous paragraph (specific to each type of sticks) a good collapse is obtained. In particular, the reduced velocity $V / V^{*}$ only varies by a factor 3 . The overall collapse seems to be less efficient regarding the reduced height but a close inspection reveals that the data splits in two separate groups, accordingly to the material used (wood or PVC). Within each of these groups, the collapse is far better (at most a factor 2 for the reduced velocity, and a factor 3.5 for the reduced height). This shows that the characteristic height and speed provide one with two accurate scales for the popsicle stick bomb.

The existence of these two sub-sets, based on the material used, can be explained and expected. The dimensional analysis used to derive the characteristic scales inherently excludes some phenomena among which two might play a crucial role: on the one hand, the solid friction between the sticks (either static or dynamic) and on the other hand, the internal dissipation. Both phenomena can be characterized by dimensionless parameters: respectively the friction coefficients $\left(\mu_{s}\right.$ and $\left.\mu_{d}\right)$ and the quality factor $Q$ (or damping) of the oscillations (see figure 3). It is clear that increasing either the friction or the damping can only lead to lower values of the height and the velocity since both solid and internal frictions are a source of energy dissipation. Performing accurate and reproducible measurements of friction coefficients can be a difficult endeavor but in our experiments it is obvious that the rough wooden popsicle sticks used have greater 
lated.
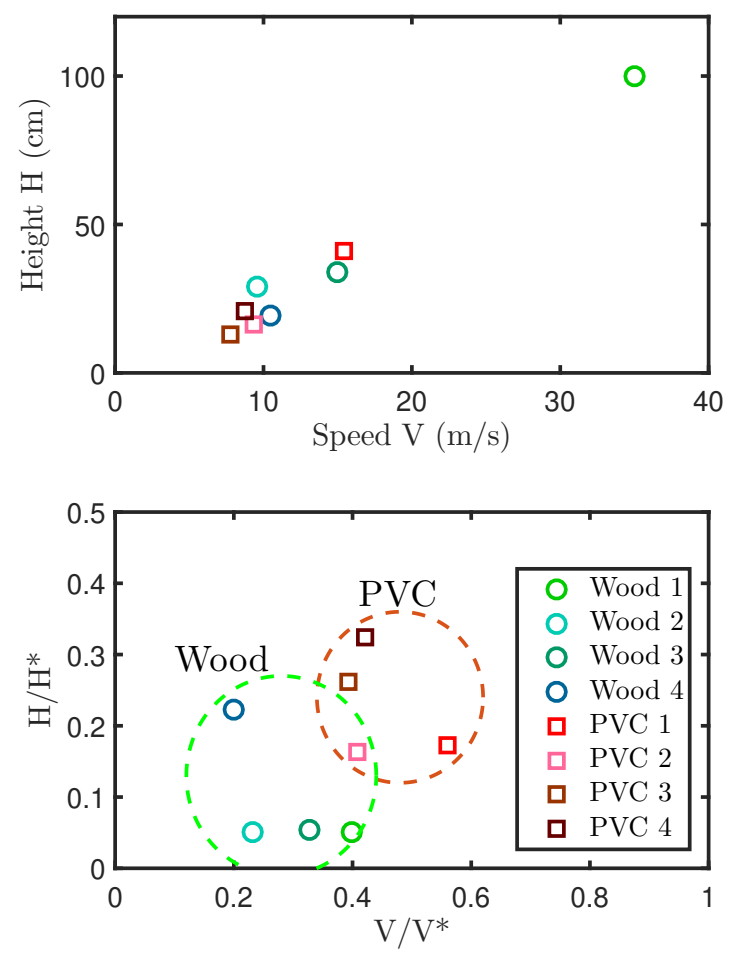

FIG. 8. Top: speed $V$ and the height $H$ of the wave for each type of sticks tested. The squares represent the four types of PVC sticks, and the circles the four types of wooden sticks. The points are widespread and no simple relationship between the two quantities is expected. Bottom: Plot of the rescaled data, $V / V *$ and $H / H *$. One can notice a good collapse of the data which gathers according to the material used.

friction coefficients than the smooth PVC sticks. Moreover, measurements of the decay rate of the free vibrations of a clamped beam clearly indicate that the internal dissipation of wood is greater than that of PVC. Altogether these observations justify the two subsets of rescaled data, and clarify why both the rescaled height and speed of wood should be less than those of PVC.

The results and conclusions presented in this paragraph can illustrate the predicting power of scaling laws. Indeed, one could have reasonably computed (within error bars) the speed and height of the stiffer dry sticks, knowing only their dimensions and the corresponding speed of sound, from the results obtained with more flexible wooden sticks. Alternatively, the height and speed of the wave of for instance thicker sticks can be extrapo-

\section{CONCLUSION}

In summary, the linear theory of elasticity allows us to derive scaling laws for the height and speed of the traveling wave. These predictions are validated through a series of experiments varying the pitch of the weave as well as the dimensions and materials of the sticks. This problem is a good example of how dimensional analysis can shed new light on a complex phenomenon.

Several aspects of the popsicle stick bomb are not addressed in this paper and might deserve further attention. It can be seen on figure 1 that the pattern is not symmetrical. Indeed, the wave differs depending on which end of the chain is released, i.e. on the direction of propagation. Equivalently, if the chain is flipped over before the release, the wave properties are affected. Note that the scaling laws should still apply although the numerical prefactors might differ. In particular, it would be interesting to study whether the rescaled velocity and height of this so-called "reverse" wave fall in the same region as those of the "regular" wave. The influence of the support has not been investigated. Indeed, the speed and height might vary whether the chain is set on a hard surface (such as a laboratory table) or on a softer surface (such as a rug or carpet). Numerous home videos found online also show that the wave can propagate without a direct support. It would therefore be interesting to study the case of a chain hanging vertically. Finally, the pattern of the weave can be varied. A cobra weave can be created by interlocking one stick to three or five (instead of four) perpendicular sticks and a square pattern can also be built. The elastic energy and characteristic time would clearly vary but once more it would be interesting to check if the scaling laws hold.

\section{ACKNOWLEDGMENTS}

The authors acknowledge support from the PALSE program of the University of Lyon Saint-Etienne, the University Lyon Claude Bernard, the Société Française de Physique and from the École Normale Supérieure de Lyon and its Physics Department. The authors are grateful to both local and international organizing committees of the International Physicists Tournament for having put together an exciting event.
* nicolas.taberlet@ens-lyon.fr

1 J. M. J. van Leeuwen, Am. J. Phys. 78, 721 (2010).

2 D. E. Shaw, Am. J. Phys. 46, 640 (1978).

${ }^{3}$ L. A. Whitehead, Am. J. Phys. 51, 182 (1983).
${ }^{4}$ H. D. Rathgeber, Am. J. Phys. 31, 62 (1963).

5 J. Higbie, Am. J. Phys. 48, 86 (1980).

6 J.-Y. Chastaing, E. Bertin, and J.-C. Géminard, Am. J. Phys. 83, 518 (2015). 
7 J.-Y. Chastaing, G. Pillet, N. Taberlet, and J.-C. Géminard, Phys. Rev. E 91, 052918 (2015).

8 D. A. Goodings and T. Szeredi, Am. J. Phys. 59, 924 (1991).

9 B. A. Lindsey, P. R. L. Heron, and P. S. Shaffer, Am. J. Phys. 80, 154 (2012).

${ }^{10}$ L. D. Landau, L. P. Pitaevskii, A. M. Kosevich, and E. M. Lifshitz, Theory of Elasticity, Third Edition: Volume 7 (Course of Theoretical Physics), 3rd ed. (ButterworthHeinemann, 1986).
11 R. D. Edge, Phys. Teach. 21, 608 (1983).

12 J. Casey, Phys. Teach. 31, 286 (1993).

13 A. B. Kaiser, Am. J. Phys. 40, 1701 (1972).

14 H. E. Huntley and J. V. Kline, Am. J. Phys. 24, 534 (1956).

15 J. Palacios and H. L. Armstrong, Am. J. Phys. 33, 513 (1965).

16 W. J. Remillard, Am. J. Phys. 51, 137 (1983).

17 T. R. Tretter, Phys. Teach. 43, 530 (2005).

18 H. D. Sheets and J. C. Lauffenburger, Phys. Teach. 37, 376 (1999). 